\title{
A rapid and low noise RANS-to-WMLES condition in curvilinear compressible ZDES simulations
}

\author{
Nicolas Renard, Sébastien Deck, and Pierre-Élie Weiss \\ ONERA The French Aerospace Lab, F-92190 Meudon, France, \\ nicolas.renard@onera.fr
}

\begin{abstract}
The rapid and low-noise strategy of Deck et al. [7] for the RANS-to-WMLES switch compatible with compressible flow solvers on curvilinear grids is presented. It can be used both as an inflow condition or as an embedded resolved turbulence injection and combines Zonal Detached Eddy Simulation, Dynamic Forcing and Zonal Immersed Boundary Conditions (for roughness elements) approaches. The relaxation length is close to 7 boundary layer thicknesses on coarse grids and the feasibility on a 3-element high-lift airfoil is demonstrated. On a flat plate, no spurious acoustic footprint of the inflow is visible in the wall pressure spectra, of which the low-frequency part is obtained. The intermittent nature of wall turbulence is captured. The hybrid RANS/LES context makes the computational effort affordable for industrial applications, e.g. aeroacoustic studies.
\end{abstract}

\section{Introduction}

Resolving wall-bounded turbulence instead of simple RANS-modelling is needed in flow situations dominated by the history of the upstream boundary layer (e.g. mild flow separation), whenever RANS models show limitations (e.g. adverse pressure gradient flows) or when the application involves a high level of description (dynamic load, aeroacoustics...). However, the cost of a Direct Numerical Simulation of wall turbulence is prohibitive and Wall-Resolved Large Eddy Simulation (WRLES) does not reduce it much because of the cost of resolving the inner layer (see estimates in [5]). As a consequence, Wall-Modelled LES is mandatory if real-life aerospace applications are treated with resolved wall turbulence.

In the hybrid RANS/LES context, a natural wall model is provided by a RANS treatment of the region closest to the wall while the outer layer of the boundary layer (e.g. $90 \%$ of its thickness) is resolved in LES. In the zonal framework provided by the Zonal Detached Eddy Simulation technique [3], this is performed in ZDES mode 3 where the interface between the near-wall RANS zone and the outer LES is set by the user. This requires more input from the user than automatic methods such as IDDES [19], but the WMLES zones and their treatment are fully under control. WMLES is employed only in the zones of 
interest, whereas the rest of the attached boundary layers is modelled in RANS, especially the thin boundary layers subject to laminar-turbulent transition. As a consequence, resolved turbulence must be injected at the upstream boundary of the WMLES domains, either embedded in the flow or at the inlet. This is the main topic of the paper.

Many strategies for resolved turbulent inflow / injection have been proposed but several difficulties must be addressed: 1) Applicability to 3D geometries, which is not easily achieved by mapping or recycling techniques such as [12] or synthetic turbulence such as $[10,15] ; 2)$ Adaptation distance, especially on the WMLES grids (coarser than for WRLES/DNS) where mapping/recycling techniques, basic tripping or vortex generators may require too large relaxation lengths; 3) Self sufficient method and initialisation, which is the case of neither mapping/recycling methods nor volumic forcing (such as [23]) alone; 4) Spurious noise which is not compatible with aeroacoustic applications, ruling out the rather noisy synthetic turbulence methods and the recycling technique which introduces a spurious frequency. A strategy simpler than the damping layer with overlapping meshes of [20] and allowing acoustic feedback is sought.

The present proposal is driven by two major criteria: 1) Applicability to 3D geometries on (somewhat coarse) WMLES grids, which implies a general curvilinear formulation capable of both inlet and embedded injection with a short relaxation length (smaller than $10-15 \delta_{0}$ ) even on coarse grids and a relative simplicity of use; 2) Low spurious noise generation. The present proposal published by Deck et al. [7] relies on three ingredients exposed in the next section. The strategy is then demonstrated on a flat plate where the wall pressure is analysed. Finally, the feasibility on a high-lift 3-element airfoil is illustrated.

\section{Proposed low-noise turbulence injection condition}

The new strategy is made of 3 ingredients: the WMLES is performed using ZDES mode 3, resolved turbulent fluctuations are generated by roughness elements represented by Zonal Immersed Boundary Conditions, and the relaxation towards sustained turbulence is accelerated by dynamic forcing.

With the first ingredient, namely ZDES mode 3, the near-wall RANS / outer LES interface is treated in a passive way by simply switching the length scale used in the Spalart-Allmaras turbulence model [22] from the wall distance in the RANS zone to the cell size estimate in the LES zone $\left(i . e . \tilde{d}=d_{w}\right.$ if $d_{w}<d_{w}^{\text {interface }}$, $\min \left(d_{w}, C_{\mathrm{DES}} \Delta_{\mathrm{vol}}\right)$ otherwise). This simple treatment is robust and does not introduce empiricism or spurious noise, but the height of the RANS/LES interface must be carefully set, independent from the mesh resolution [6] (which means there is no meshing constraint, unlike other methods) and at an outer-scaled position, typically $d_{w}^{\text {interface }} / \delta=0.1[5]$ (which requires preprocessing). Further refinement is proposed in [16] but not crucial here.

The second ingredient generates resolved fluctuations by means of properly scaled roughness elements. Their rationale relies on the literature on non-normal linear stability of turbulent boundary layers. Especially Cossu et al. [2] showed 
the existence of optimal transient growth initiated by outer-scaled streamwise vorticity perturbations, in addition to inner-scaled ones. The cylindrical obstacles inserted in the simulation are devised to produce perturbations similar to the outer-scaled optimal perturbations (i.e. highly amplified) indicated by [2]. They are described by Zonal Immersed Boundary Conditions inspired by classical methods which have been adapted to hybrid RANS/LES methods [24]. Figure 1 summarizes the geometrical parameters of each test case considered in the following and illustrates how the roughness elements generate resolved fluctuations, by creating horse-shoe vortices which provide large-scale streamwise vorticity (a key feature of optimal perturbations) with resulting hairpin vortices clearly visible.

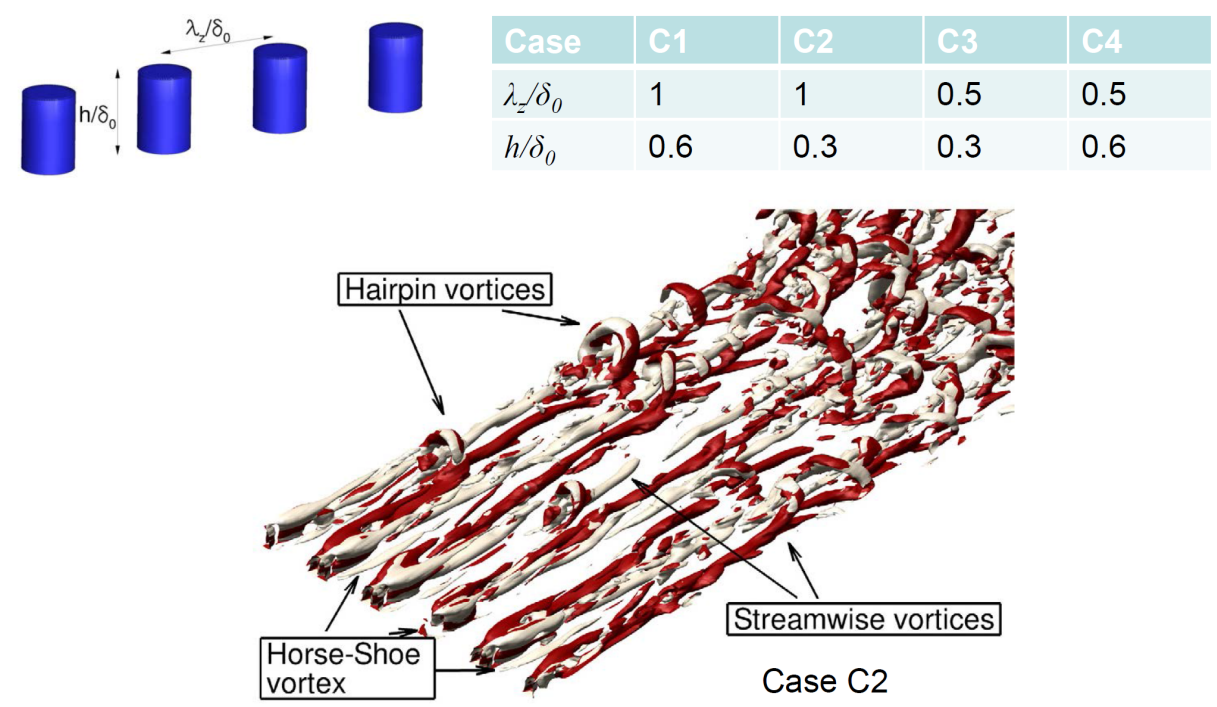

Fig. 1. Geometrical parameters of the roughness elements for cases C1 to C4. Zoom near the resolved turbulence injection (case $\mathrm{C} 2$ ) : iso surface of the $\mathrm{Q}$ criterion $Q=$ $0.25 U_{0}^{2} / \delta_{0}^{2}$ coloured by the streamwise component of vorticity $\left(\omega_{x}<0\right.$ in red and $\omega_{x}>0$ in white).

The injected resolved fluctuations turn quickly into physical turbulence because the third ingredient is used, namely dynamic forcing. This strategy to decrease the relaxation length is inspired by the pioneering work of Spille-Kohoff \& Kaltenbach [23], specifically adapted to ZDES by Laraufie et al. [11] and formulated in general curvilinear cases in [4]. The forcing relies on a proportional controller and on conditions to focus the action on the more energetic events and to prevent from unrealistic large shear stress events. The target Reynolds stresses are estimated from a prior RANS computation. 
The proposed strategy coupling all 3 ingredients is assessed in the following on a flat plate and on a high-lift 3-element airfoil.

\section{Demonstration of the new strategy on a flat plate}

The test case considered here and sketched in fig. 2 is a flat-plate spatially growing turbulent boundary layer with zero pressure gradient, $M_{0}=0.21$ and $3040 \leq R e_{\theta} \leq 6100$. The mesh has a streamwise spacing $\Delta x / \delta$ close to 0.1 $\left(\Delta x^{+}=100-200\right)$ and a spanwise spacing $\Delta z^{+}=50$, counting $7.7 \cdot 10^{6}$ points. The RANS/LES interface is set at $0.125 \delta$ from the wall. The present strategy (cases C, with 4 different parameter cases, see fig. 1) is compared with more classical inflow methods: cases A resort to the Synthetic Eddy Method [15], without (A1) and with (A2) dynamic forcing to accelerate relaxation, and case B involves White Noise injection together with dynamic forcing.

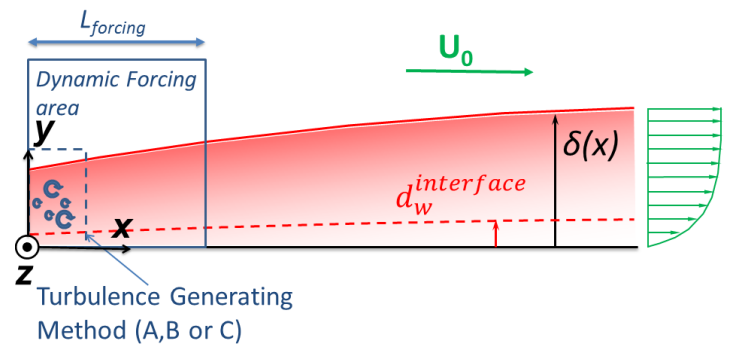

Fig. 2. Sketch of the flat-plate turbulent boundary layer test case.

The instantaneous flow-field is visualized in fig. 3. The improvement of the relaxation length provided by the dynamic forcing is illustrated by the comparison between the SEM cases (A1 and A2). Furthermore, even with a basic white noise injection (case B), coherent structures are quickly generated thanks to the dynamic forcing. The density variations plots also reveal that the classical inflow methods ( $\mathrm{A}$ and $\mathrm{B}$ ) induce a significant spurious noise, contrary to the present strategy (cases C) where the relaxation process looks satisfying from the $\mathrm{Q}$ criterion perspective.

The performance of the strategies is quantitatively assessed in fig. 4 where the convergence of the skin friction towards its physical value is depicted. In addition to several datasets available in the literature including both DNS and experimental data $([14,8,18,21])$, numerical results are also compared with the widely acknowledged Coles-Fernholz correlation calibrated by Nagib et al[13]. The essential role of the dynamic forcing is confirmed and acceptable results are obtained even with the basic white noise injection. The present strategy provides a short relaxation length, as small as $7 \delta_{0}$ in case $\mathrm{C} 4$ (which coincides with the forcing distance). After the relaxation, the mean velocity and turbulence 


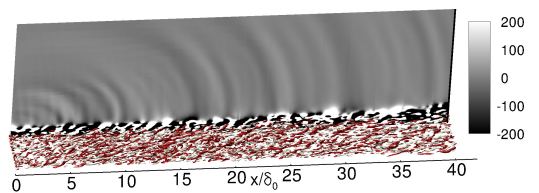

(a) case A1

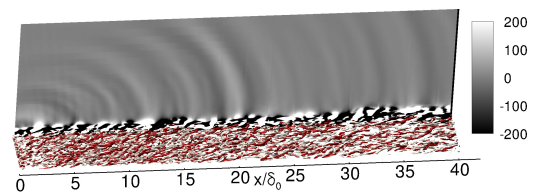

(b) case A2

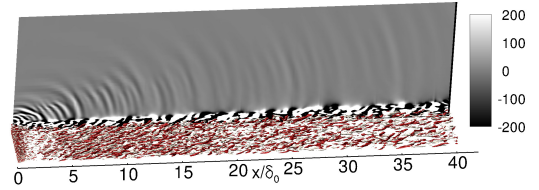

(c) case B

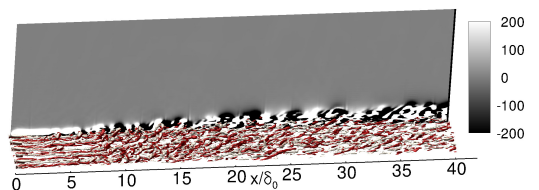

(d) case $\mathrm{C} 1$

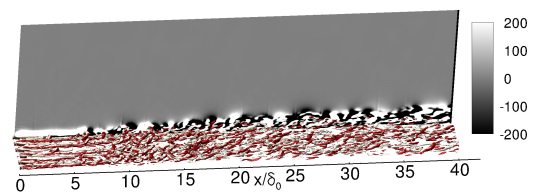

(e) case $\mathrm{C} 2$

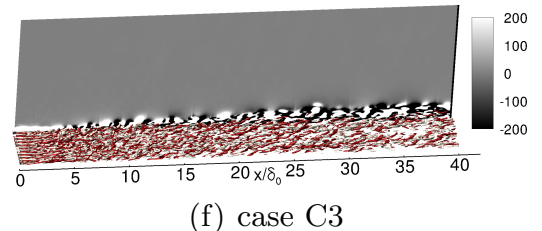

Fig. 3. Iso surface of the $\mathrm{Q}$ criterion $Q=0.25 U_{0}^{2} / \delta_{0}^{2}$ coloured by the streamwise component of vorticity $\left(\omega_{x}<0\right.$ in red and $\omega_{x}>0$ in white). Instantaneous field of $-\frac{\partial \rho}{\partial t}$ (gray scale colormap). 
intensity profiles converge to very similar values, as illustrated at $R_{\theta}=5200$ in fig. 5 where the ZDES computations are compared with experimental and DNS data. Well-known limitations of hybrid RANS/LES methods for WMLES such as ZDES mode 3 are also visible, namely a deviation of the mean velocity with respect to the logarithmic law near the RANS/LES interface and an underestimation of the streamwise turbulence intensity at an intermediate height in the outer layer. These issues are related to the WMLES rather than to the inflow condition and would be less visible at higher Reynolds numbers. They are not treated in this paper. The contributions of the resolved fluctuations to the Reynolds shear stress is depicted in fig. 6 in case C3, showing that fluctuations do penetrate through the RANS/LES interface down to the wall. The consequences on the resolution of mean skin friction are discussed in [17].

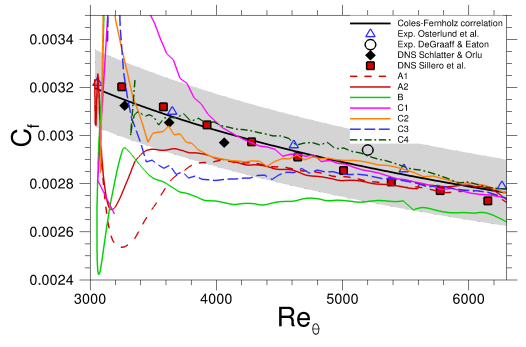

(a) $C_{f}\left(R e_{\theta}\right)$

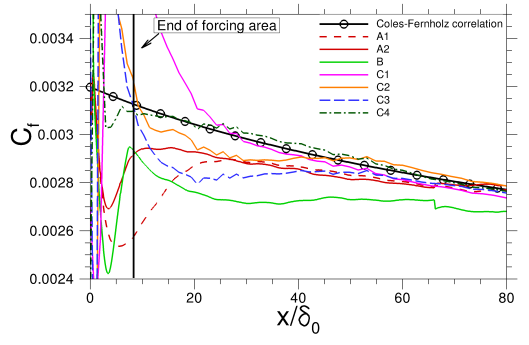

(b) $C_{f}\left(x / \delta_{0}\right)$

Fig. 4. Streamwise evolution of the skin friction coefficient. The shaded area depicts a $5 \%$ tolerance margin with respect to the Coles-Fernholz correlation $C_{f}^{C F}=$ $2\left(\frac{1}{0.384} \ln \left(R e_{\theta}\right)+4.127\right)^{-2}[13]$.

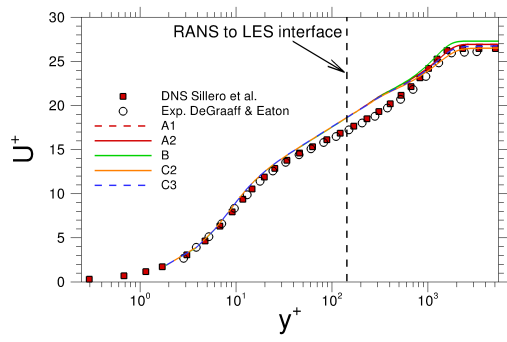

(a) Mean velocity profile

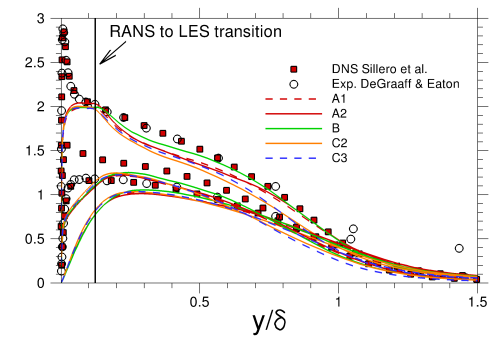

(b) Resolved normal Reynolds stresses $\left(u_{r m s}^{+}, v_{r m s}^{+}, w_{r m s}^{+}\right)$

Fig. 5. Reynolds-averaged data at $R e_{\theta}=5200$ 


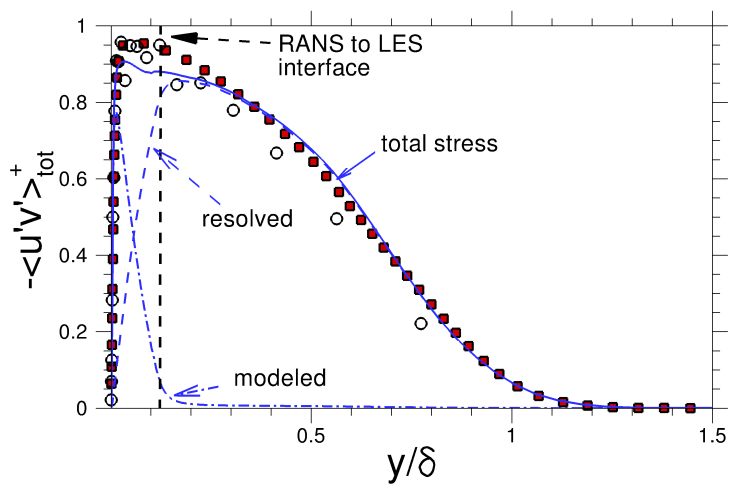

Fig. 6. Decomposition of the total Reynolds shear stress into resolved and modelled parts on configuration $\mathrm{C} 3$ at $\operatorname{Re}_{\theta}=5200$.

The acoustic footprint of the inflow methods is assessed in fig. 7. The comparison of the $p_{r m s}$ profiles with DNS data clearly shows that the pressure field in the computations with SEM or White Noise conditions includes spurious noise from the inflow, although the $R e_{\theta}=5200$ station is far downstream from it. On the contrary, the present strategy underestimates $p_{r m s}$ compared with DNS, which makes sense since turbulence is only partially resolved. The wall pressure Power Spectral Density (PSD) is assessed and compared with the Goody model [9]. The spectra feature a spurious content at high frequencies with SEM or White Noise inflow conditions, with higher frequencies for White Noise, which may be explained by the little spatio-temporal correlation of the fluctuations created by White Noise involving smaller scales than SEM does. Conversely, the calculations with the present strategy do not show spurious spectral content at high frequencies, and the low-frequency range of the spectrum is in reasonable agreement with the Goody model in spite of some under-prediction at low frequencies. In the WMLES context, the fact that the high-frequency range of the spectrum is missing is expected. Consequently, the predictions of wall pressure spectra in cases C2 and C3 may be considered as satisfactory. If the trend towards higher Reynolds numbers is considered, based on the work of [1], one may expect that the present approach may predict the lower-frequency part of the wall pressure spectrum so that half of the wall pressure variance could be resolved at very high Reynolds numbers.

The wall pressure signals are further investigated in fig. 8 where their Probability Density Function is evaluated and compared to experimental data. The main finding is that when the wall pressure signal is dominated by spurious noise from the inflow, it has a quasi-Gaussian distribution (consistent with the central limit theorem), while in the present strategy, the distribution of the wall pressure fluctuations includes more frequently strong events (the tails of the distribution are heavier than for a Gaussian distribution), which is known to be related to 


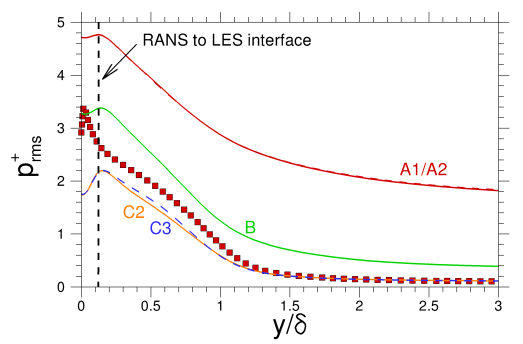

(a) Wall-normal distribution of pressure $\operatorname{rms} p_{r m s}^{+}=\frac{p_{r m s}}{\rho u_{\tau}^{2}}$

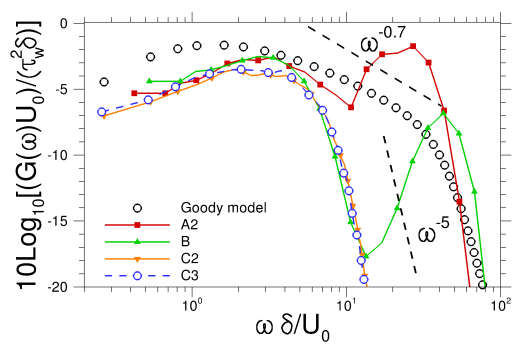

(b) PSD of wall pressure fluctuations

Fig. 7. Pressure fluctuations at $R e_{\theta}=5200$

the intermittency of turbulence. The good match of the PDF of wall pressure with the experiment suggests that the physical features related to the intermittent nature of turbulence are properly reproduced at the wall with the present strategy and not dominated by spurious noise from the inlet.

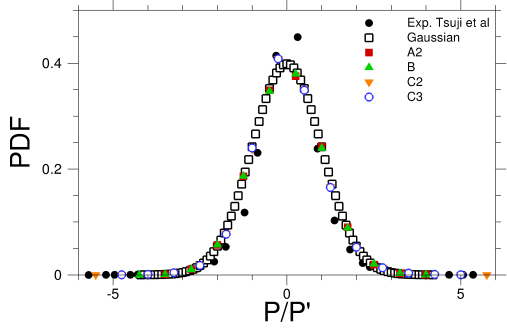

(a) linear scales

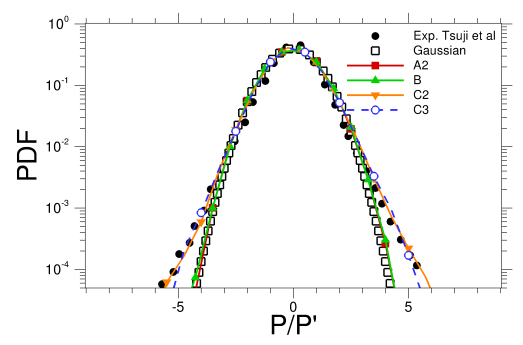

(b) semilogarithmic scales

Fig. 8. PDF of wall pressure fluctuations normalized by their root mean squared value at $R e_{\theta}=5200$.

\section{Feasibility on a high-lift 3-element airfoil}

The present strategy is applied to a high-lift 3-element airfoil to demonstrate its feasibility and robustness for industrial flow problems with curvilinear geometries and resolved turbulence injection (embedded in the numerical domain rather than as inlet condition, so that a possible acoustic feedback propagating through the injection area is allowed). Each zone of the flow is treated with the appropriate mode of the ZDES technique, as detailed in fig. 9. ZDES mode 3 is used only where needed, i.e. near the trailing edge of the main element (providing 
a proper resolved turbulence to the interaction of the wake of the main element with the flow above the flap). A very short relaxation length is needed between the resolved turbulence injection and the trailing edge. The present strategy is used with parameters analogous to case C3 in the flat plate tests where it provides a short skin friction relaxation distance and low spurious noise.

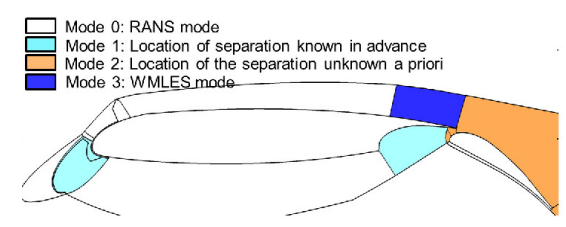

(a) ZDES zones for the calculation of the 3-element airfoil

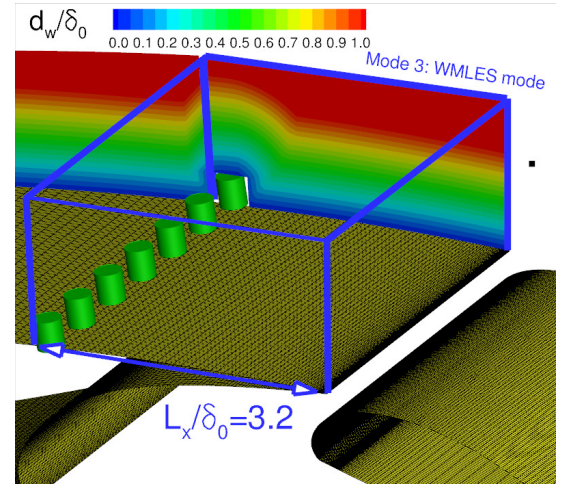

(b) Roughness elements in the WMLES domain similar to Case C3 (see fig. 1).

Fig. 9. Computational description. $d_{w} / \delta_{0}$ is the normalized distance to the wall where $\delta_{0}$ is the boundary layer thickness at the inlet domain.

The chord Reynolds number is $R e_{c}=2.09 \cdot 10^{6}$, the free-stream Mach number $M_{0}=0.15$, and the mesh counts $50 \cdot 10^{6}$ points. It should be noted that a WRLES of the same problem would have required several billions points in contrast, which demonstrates the beneficial influence of the hybrid RANS/LES context. As illustrated by fig. 10 and 11, the present strategy succeeds in generating quickly a realistic resolved turbulence content near the trailing edge of the main element and causes much less spurious noise than the White Noise approach. These results suggest that the low-noise strategy can be applied to more complex geometries.

\section{Outlook}

A rapid and low-noise strategy for RANS-to-WMLES switch on curvilinear grids and compressible flow solver has been introduced for both inflow and embedded injection of resolved turbulence, as published by Deck et al. [7]. It combines the Zonal Detached Eddy Simulation technique with a Dynamic Forcing relaxation enhancement and a Zonal Immersed Boundary Condition description of roughness elements. The relaxation distance on relatively coarse grids typical of WMLES is as small as $7 \delta_{0}$ with properly chosen geometrical parameters for the 


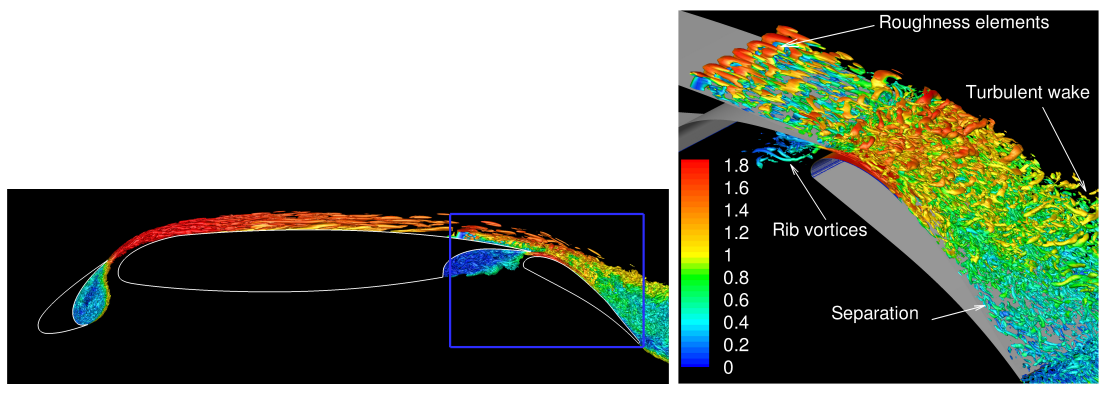

Fig. 10. Isosurface of the $Q$ criterion coloured by the velocity magnitude $|\underline{u}| / U_{0}$ in the flap area (top view: $Q \frac{c^{2}}{U_{0}^{2}}=100$; zoom: $Q \frac{c^{2}}{U_{0}^{2}}=3000$ ).

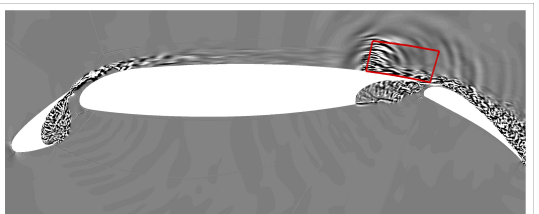

(a) ZDES mode 3 - anisotropic White Noise - Dynamic Forcing (similar to Case B)

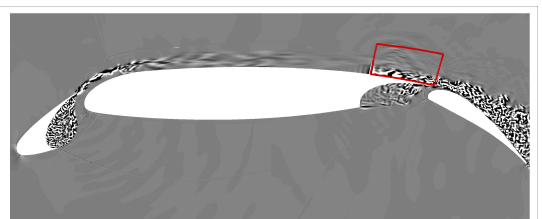

(b) ZDES mode 3 - ZIBC - Dynamic Forcing (similar to Case C3)

Fig. 11. Instantaneous field of $-\frac{1}{\rho} \frac{\partial \rho}{\partial t}$. 
obstacles, following the non-normal linear stability analysis results in the literature. The feasibility on a more complex geometry, namely a 3-element high-lift airfoil, has been demonstrated. The analysis of the pressure signals in a flat-plate turbulent boundary layer with zero pressure gradient indicates that no spurious acoustic footprint from the inlet condition is visible, contrary to the classical approaches considered here (synthetic turbulence or white noise injection). The low-frequency part of the wall pressure spectrum is obtained and it is expected that half of the wall pressure variance may be obtained in such WMLES computations at very high Reynolds numbers. Moreover, the intermittency of the signal, related to the nature of turbulence, is captured.

The present approach has potential for aeroacoustic studies (e.g. jet, trailing edge, boundary layer or cabin noise), all the more as the hybrid RANS/LES context makes the computational effort affordable for industrial applications.

\section{Acknowledgments}

The authors wish to thank all the people involved in the past and present evolution of the FLU3M code. The ZIBC approach has been developed in the framework of the research project ALLIGATOR funded by ONERA. Part of this work is also related to the EU collaborative research project Go4Hybrid, funded by the European Community in the 7th Framework Programme, under Contract No. 605361.

\section{References}

1. Aupoix, B.: Extension of Lysak's Approach to Evaluate the Wall Pressure Spectrum for Boundary Layer Flows. Flow Turbulence and Combustion, vol 94, No. 1, pp 63-78 (2015)

2. Cossu, C., Pujals, G., Depardon, S.: Optimal transient growth and very largescale structures in turbulent boundary layers. Journal of Fluid Mechanics 619, 79-94 (2009)

3. Deck, S.: Recent improvements in the Zonal Detached Eddy Simulation (ZDES) formulation. Theoretical and Computational Fluid Dynamics 26, 523-550 (2012)

4. Deck, S., Laraufie, R.: Numerical investigation of the flow dynamics past a threeelement aerofoil. Journal of Fluid Mechanics 732, 401-444 (2013)

5. Deck, S., Renard, N., Laraufie, R., Sagaut, P.: Zonal Detached Eddy Simulation (ZDES) of a spatially developing flat plate turbulent boundary layer over the Reynolds number range $3150 \leq R e_{\theta} \leq 14000$. Physics of Fluids 26, 025,116 (2014)

6. Deck, S., Weiss, P.E., Pamiès, M., Garnier, E.: Zonal Detached Eddy Simulation of a spatially developing flat plate turbulent boundary layer. Computers \& Fluids 48, 1-15 (2011)

7. Deck, S., Weiss, P.E., Renard, N.: A rapid and low noise switch from RANS to WMLES on curvilinear grids with compressible flow solvers. Journal of Computational Physics 363, 231-255 (2018)

8. DeGraaff, D.B., Eaton, J.K.: Reynolds number scaling of the flat plate turbulent boundary layer. Journal of Fluid Mechanics 422, 319-346 (2000) 
9. Goody, M.: Empirical Spectral Model of Surface Pressure Fluctuations. AIAA J. vol.42, No.9, pp 1788-1794 (2004)

10. Jarrin, N., Prosser, R., Uribe, J.C., Benhamadouche, S., Laurence, D.: Reconstruction of turbulent fluctuations for hybrid RANS/LES simulations using a SyntheticEddy Method. International Journal of Heat and Fluid Flow 30, 435-442 (2009)

11. Laraufie, R., Deck, S., Sagaut, P.: A dynamic forcing method for unsteady turbulent inflow conditions. Journal of Computational Physics 230, 8647-8663 (2011)

12. Lund, T.S., Wu, X., Squires, K.D.: Generation of turbulent inflow data for spatially-developing boundary layer simulations. Journal of Computational Physics 140, 233-258 (1998)

13. Nagib, H.M., Chauhan, K.A., Monkewitz, P.A.: Approach to an asymptotic state for zero pressure gradient turbulent boundary layers. Philosophical Transactions of the Royal Society A 365, 755-770 (2007)

14. Österlund, J.M., Johansson, A.V., Nagib, H.M., Hites, M.H.: A note on the overlap region in turbulent boundary layers. Physics of Fluids 12(1), 1-4 (2000)

15. Pamiès, M., Weiss, P.E., Garnier, E., Deck, S., Sagaut, P.: Generation of synthetic turbulent inflow data for large eddy simulation of spatially evolving wall-bounded flows. Physics of Fluids 21, 045,103 (2009)

16. Renard, N., Deck, S.: Improvements in Zonal Detached Eddy Simulation for Wall Modeled Large Eddy Simulation. AIAA Journal 53(11), 3499-3504 (2015). DOI 10.2514/1.J054143

17. Renard, N., Deck, S.: On the resolution of mean skin friction by hybrid RANS/LES simulations at high Reynolds numbers. In: S. et al. (ed.) Direct and Large-Eddy Simulation XI, pp. 367-372. Springer (2019)

18. Schlatter, P., Örlü, R.: Assessment of direct numerical simulation data of turbulent boundary layers. Journal of Fluid Mechanics 659, 116-126 (2010)

19. Shur, M.L., Spalart, P.R., Strelets, M.K., Travin, A.K.: A hybrid RANS-LES approach with delayed-DES and wall-modelled LES capabilities. International Journal of Heat and Fluid Flow 29, 1638-1649 (2008)

20. Shur, M.L., Spalart, P.R., Strelets, M.K., Travin, A.K.: Synthetic turbulence generators for RANS-LES interfaces in zonal simulations of aerodynamic and aeroacoustic problems. Flow Turbulence and Combustion 93, 63-92 (2014)

21. Sillero, J., Jimenez, J., Moser, R.: One-point statistics for turbulent wall-bounded flows at Reynolds numbers up to $\delta^{+} \approx 2000$. Physics of Fluids 25, 105,102 (2013)

22. Spalart, P.R., Allmaras, S.R.: A one-equation turbulence model for aerodynamic flows. La Recherche Aérospatiale (1), 5-21 (1994)

23. Spille-Kohoff, A., Kaltenbach, H.: Generation of turbulent inflow data with a described shear-stress profile. In Proceedings pp 137-147, third AFOSR Int. Conf. on DNS/LES, Arlington, edited by C.Liu, L. Sakell and T. Beutner, Greyden press, Columbus, OH, 5-9 August (2001)

24. Weiss, P., Deck, S.: On the coupling of a zonal body-fitted/immersed boundary method with ZDES: application to the interactions on a realistic space launcher afterbody flow. Computers \& Fluids, in press, doi:10.1016/j.compfluid.2017.06.015 (2017). DOI https://doi.org/10.1016/j.compfluid.2017.06.015 